\title{
Attentional modulation of visual-evoked potentials by threat: Investigating the effect of evolutionary relevance
}

\author{
Christopher Brown ${ }^{\mathrm{a}}$, Wael El-Deredy ${ }^{\mathrm{b}}$, Isabelle Blanchette ${ }^{\mathrm{c}, *}$ \\ ${ }^{a}$ Human Pain Research Group, University of Manchester, UK \\ ${ }^{\mathrm{b}}$ School of Psychological Sciences, University of Manchester, UK \\ ${ }^{\mathrm{c}}$ Département de Psychologie, Université du Québec à Trois-Rivières, Canada
}

\section{A R T I C L E I N F O}

\section{Article history:}

Accepted 21 August 2010

\section{Keywords:}

Threat

Attention

P1

ERP

Evolution

Fear module

Visual attention

\begin{abstract}
A B S T R A C T
In dot-probe tasks, threatening cues facilitate attention to targets and enhance the amplitude of the target P1 peak of the visual-evoked potential. While theories have suggested that evolutionarily relevant threats should obtain preferential neural processing, this has not been examined empirically. In this study we examined the effects of evolutionarily relevant (e.g. spiders and snakes) and irrelevant (e.g. knifes and syringes) threatening cues. Non-predictive threatening cues (in left or right visual field) were paired with non-threatening cues and were followed by neutral targets in the same or opposite location. The amplitude of the target P1 was increased in contralateral electrodes when the target followed in the same location as the threatening cues. This effect did not interact with evolutionary relevance. Both evolutionary relevant and evolutionary irrelevant threats led to increased P1 amplitude, although the effect was stronger for modern threats. We conclude that the threat-superiority effect is robust and largely independent of the type of threatening stimulus.
\end{abstract}

(c) 2010 Elsevier Inc. All rights reserved.

\section{Introduction}

Threatening stimuli associated with fear or danger are powerful at capturing attention relative to non-threatening stimuli, a phenomenon known as the threat-superiority effect (Blanchette, 2006; Öhman, Flykt, \& Esteves, 2001; Öhman, Lundqvist, \& Esteves, 2001; Smith, Cacioppo, Larsen, \& Chartrand, 2003). Results from behavioural studies show that threatening stimuli (e.g. snakes or spiders) are more quickly and efficiently responded to than neutral stimuli (Öhman \& Mineka, 2001; Öhman et al., 2001), and that this effect is more pronounced in individuals with specific phobias of threatening animals, or in individuals with high levels of trait anxiety (Li, Li, \& Luo, 2005).

Faster detection of threatening stimuli is commonly interpreted within an evolutionary framework, in which survival advantages are conferred by fast detection of recurring threats. An influential theory of the neural circuitry involved has posited the existence of a 'fear module' in the brain that evolved prior to the cerebral cortex (Öhman, Flykt, Lundqvist, Lane, \& Nadel, 2000; Öhman \& Mineka, 2001). This fear module would constantly monitor the environment for threat and initiate automatic responses (i.e. in

\footnotetext{
* Corresponding author. Address: Département de Psychologie, Université du Québec à Trois-Rivières, C.P. 500, Trois-Rivières (Québec), Canada G9A 5H7. Fax: +1 8193765195.

E-mail address: isabelle.blanchette@uqtr.ca (I. Blanchette).
}

parallel to cortical processing). In this model, the fear module should be best triggered by threats that were relevant at the time when common aspects of the mammalian brain evolved (e.g. threatening animals) (Öhman et al., 2000). More generally, a strong evolutionary thesis would suggest that not only are the mechanisms of fear detection constrained by evolution, but that the specific triggers of these mechanisms should also be highly constrained. A weaker evolutionary view might propose that while the neural pathways underlying threat detection may have been shaped by evolution, the specific triggers for activation of these pathways may reflect ontogenetic contingencies.

Recent empirical research using reaction time data shows that although biological threats are commonly used to explore the attentional mechanisms of threat processing, the threat-superiority effect is not limited to stimuli that have evolutionary significance. Modern threats that are not part of evolutionary history (e.g. knives and syringes) are detected just as rapidly and efficiently as biological threats that are evolutionarily relevant (Blanchette, 2006; Brosch \& Sharma, 2005; Fox, Griggs, \& Mouchlianitis, 2007) and that children will show a threat-superiority effect for evolutionarily irrelevant fear objects that they have had experience with, such as syringes (LoBue, 2010). This supports the conclusion that ontogenetic contingencies can affect the threat-superiority effect.

While this data shows similar behavioural outcomes for evolutionarily relevant and irrelevant threats, it is not clear whether these outcomes are the product of the same or distinct neural 
mechanisms. One possibility is that similar reaction times result from the processing of evolutionarily recent and ancient targets by separate neural and cognitive processes. Another possibility is that both types of threats are processed through the same mechanism. In this paper, we report an experiment that attempts to address this question directly using event-related potentials (ERPs).

Previous studies have examined the neural processing of threatening stimuli generally, compared to neutral stimuli. For instance, studies have shown increased activation in the visual cortex to threatening vs. neutral stimuli (Lane, Chua, \& Dolan, 1999; Pourtois, Schwartz, Seghier, Lazeyras, \& Vuilleumier, 2006). With their higher temporal resolution, electroencephalographic (EEG) recordings of event-related brain potential (ERPs) have also been used to compare attentional processing of threatening and non-threatening stimuli. Studies have shown that the amplitude of the P1 peak of the visual ERP to neutral target stimuli is enhanced by prior presentation of threatening relative to neutral cues (Pourtois, Thut, De Peralta, Michel, \& Vuilleumier, 2005; Pourtois et al., 2006), and that this effect is greater in individuals with high levels of trait anxiety (Li et al., 2005). This is consistent with the fact that the amplitude of the P1 peak, widely interpreted to represent early processing in visual pathways, varies as a function of the amount of attention allocated to the stimulus being processed (Clark \& Hillyard, 1996).

This work suggests that not only does threat modulate neural processing but that it does so very rapidly. There is converging evidence supporting this conclusion. For instance single cell recording in the visual cortex shows modulation of neuronal activity by emotional expression starting around $100 \mathrm{~ms}$ post-stimulus in monkeys (Sugase, Yamane, Ueno, \& Kawano, 1999). Recent work is starting to uncover the neuroanatomical pathways underlying such early modulation, in particular illustrating the links between the amygdala and visual sensory areas. Anatomical studies in primates as well as tractography studies in humans show important efferent connections from the amygdala to all levels of the ventral visual processing stream, including striate and extrastriate sites (Amaral, Behniea, \& Kelly, 2003; Catani, Jones, Donato, \& Ffytche, 2003). An fMRI study of patients with amygdala lesions showed that such damage eliminated the modulation of visual cortex activity by facial expression observed in control participants, confirming a link between threat detection in the amygdala and sensory processing in visual cortex (Vuilleumier, Richardson, Armony, \& Driver, 2004). Some hypothesize that the modulation of visual cortex activity could occur early, before object recognition, as it relies on the processing of coarse, low spatial frequency information processed by magnocellular pathways (Vuileumier, Armony, Driver, \& Dolan, 2003). Thus, threat detection in the amygdala may rapidly modulate sensory processing in visual cortex, which would be reflected in a modulation of the P1, thought to be linked with processing in extrastriate areas. Consistent with this, Pourtois, Dan, Grandjean, Sander, and Vuilleumier (2005) have shown that the modulation of the P1 by threat remained when high spatial frequency information was filtered out from visual stimuli, but not when low spatial frequency information was filtered out, and that the source of this effect was the extrastriate visual cortex. Furthermore, one study has also shown that patients with amygdala damage show a reduced or eliminated modulation of P1 by fearful faces (Rotshtein et al., 2010 cited in Vuilleumier \& Driver, 2007).

All these studies provide the neuroanatomical and neurofunctional evidence for the threat-superiority effect, and confirm that there are pathways that result in rapid modulation of neural processing by threat. No study to date has however compared the neurophysiological trace of the threat-superiority effect as a function of the evolutionary significance of the stimuli.

In this study, we compared the ERPs evoked by evolutionary relevant and irrelevant threats, focusing on the P1 peak. We used the dot-probe task (Posner, Walker, Friedrich, \& Rafal, 1984), which has been widely used to study attentional effects on ERPs (Perchet, Revol, Fourneret, Mauguiere, \& Garcia-Larrea, 2001; Perez-Edgar, Fox, Cohn, \& Kovacs, 2006; Rich et al., 2005), and the effect of threat on ERPs (Pourtois et al., 2006). In this task, a target appears in one of two locations and requires a speeded response. Prior to the target, two cues are presented simultaneously, one threatening and one neutral, in each of the two possible locations. Responses to targets are typically faster when the target follows in the same location as the threatening cue (congruent trials), as opposed to when the target follows in the location of the neutral cue (incongruent trials) an effect widely interpreted to indicate increased attention to the threatening targets (Pourtois, Thut, et al., 2005). Moreover, as mentioned previously, the amplitude of the P1 peak of the visual ERP to the target is enhanced on congruent trials, when the target follows in the same location as the threatening cue (Pourtois, Thut, et al., 2005; Pourtois et al., 2006).

Our first hypothesis was that the visual P1 peak generated in response to targets would be enhanced by pre-target threatening cues relative to neutral cues, generally. The novel contribution of this study is to explore whether the amplitude and latency of P1 peak to targets is differentially affected by evolutionarily relevant and irrelevant threat cues. We reasoned that if the strong evolutionary thesis is correct, processing would be facilitated by evolutionarily relevant vs. irrelevant threats via different neural mechanisms. This may be reflected in different modulation of the amplitude or the latency of the P1 component by evolutionary relevant and irrelevant threats, compared to their neutral non-threatening counterparts. Conversely, if evolutionary constraints on the detection of threats do not act on the triggers, but only on the mechanisms underlying threat detection, attentional facilitation by the two types of threat would be associated with similar patterns of neural activation. This would result in similar latency and amplitude of the P1 peak.

\section{Method}

\subsection{Participants}

Thirty-two healthy, right-handed individuals participated in the study. Participants gave informed written consent and the study was approved by the School of Psychological Sciences ethics committee at the University of Manchester. All participants had normal, or corrected to normal, vision, were free from any phobias and did not have any history of psychiatric or neurological illness. Participants received course credits for their participation.

\subsection{Experimental stimuli}

The experiment included two types of visual stimuli, presented in the following order on each trial: cues and targets. The stimuli were presented on a $10.5^{\prime \prime} \times 8^{\prime \prime}$ colour monitor. The cue stimuli consisted of threatening/neutral picture pairs, examples of which are presented in Fig. 1a, either side of a fixation cross. Two types of threatening cues were used, evolutionary relevant and irrelevant. Evolutionary relevant cues (snakes and spiders) were paired with neutral cues (ropes and trees respectively) of similar shapes and visual complexity in terms of orientation, brightness and level of detail. Evolutionary irrelevant threatening cues (knives and syringes) were also paired with neutral cues of similar shape and equal visual complexity (spoons and pens respectively). The threatening stimuli were shown in the left visual field in 50\% of the trials and in the right visual field in the other 50\%, and were presented in the lower visual quadrants (viewing distance $70 \mathrm{~cm}$ ) in order to have the visual stimuli and the fingers in the visual field (see Eimer, 1993). 
a

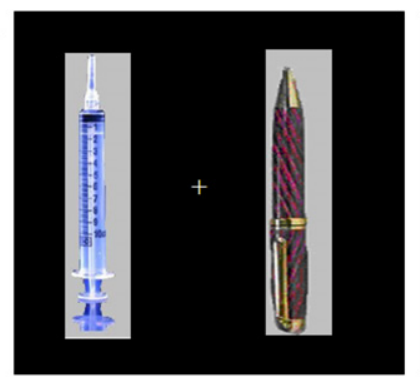

b
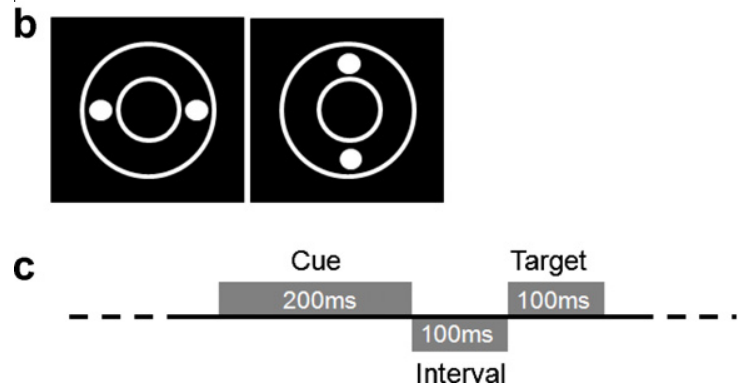

Fig. 1. Experimental stimuli. (a) Examples of the paired stimuli presented as threat cues, containing either evolutionarily irrelevant stimuli (left box) or evolutionarily relevant stimuli (right box). (b) The two different targets used, which required different responses from participants. (c) The timings of threat cue and target presentation on each experimental trial.

Target stimuli consisted of pairs of dots placed within a circle, and appeared in the location previously occupied by one of the cues. Two types of target stimuli could be presented on each trial; the pairs of dots were placed either horizontally or vertically (Fig. 1b). Participants were randomly selected to respond to the horizontal or vertical targets with equal numbers of participants responding to each. Targets were presented an equal number of times on the left and the right sides. The ratio of targets to non-targets was 1:4. The EEG data was analyzed only on non-target trials, when participants did not make a response. The study was a $2 \times 2 \times 2$ design, with trials that differed according to the type of cues (evolutionary relevant vs. irrelevant), the position of the threat cue (left vs. right) and the position of the target (left vs. right).

\subsection{Experimental procedure}

A trial began with the presentation of a cue pair for $200 \mathrm{~ms}$ After a further interval of $100 \mathrm{~ms}$ in which the screen remained blank, a target appeared at the location of one of the cues for $100 \mathrm{~ms}$ (Fig. 1c). Participants were instructed to respond to the target stimulus as quickly as possible by pressing the ' 1 ' key on a computer keyboard if the target appeared on the left of the screen or the 'backspace' key if it appeared on the right. Inter-trial intervals were randomised around $1000 \mathrm{~ms}(+15 \mathrm{~ms},-15 \mathrm{~ms})$. Each of the four types of cue pairs (evolutionary relevant vs. irrelevant cues, target presented on the left vs. the right side) appeared 48 times in a randomised order, resulting in a total of 192 trials. Participants were instructed to remain focused on the cross that was permanently presented between the two locations throughout the entire experiment in order to minimize eye movements, and these instructions were repeated after each block.

\subsection{Electroencephalographic (EEG) recordings}

During the stimulus presentations, EEG recordings were taken from $61 \mathrm{Ag} / \mathrm{AgCl}$ scalp electrodes placed according to an extended 10-20 system (Neuroscan, Inc.). We used a sampling rate of $500 \mathrm{~Hz}$ and gain of 500. Electrode FPz was used as the ground electrode, whereas electrode $\mathrm{Cz}$ was used as the reference. Vertical and horizontal electro-oculograms (EOG) were measured from bipolar channels for off-line reduction of blink and eye-movement artefacts. The impedance of all scalp electrodes was kept below $5 \mathrm{k} \Omega$.

Bandpass filters were set at $2-20 \mathrm{~Hz}$, because the P1 was of particular interest and that this peak is linked to a frequency of about $10 \mathrm{~Hz}$. Using a high pass filter of $2 \mathrm{~Hz}$ in particular was used to reduce the influence of the previous cue P2 on the target P1 which was of interest.

\subsection{Behavioural data analysis}

Reaction time data from participants' button-press responses to the target stimuli were averaged for each condition separately. In order to determine the effects of evolutionary relevant and irrelevant threats on reaction times to the targets, the data was entered into a repeated measures ANOVA $(n=31)$ consisting of three factors (cue type, threat cue side, target side).

\subsection{EEG data analysis}

The data was re-referenced to a common average (excluding $\mathrm{Cz}$, $\mathrm{FPz}$ and the EOG channels). Eye blinks and mains interference were removed using independent components analysis according to Jung et al. (2000). The data were epoched into single trials of $1.4 \mathrm{~s}$ duration starting $200 \mathrm{~ms}$ before cue onset and ending $1200 \mathrm{~ms}$ after stimulus onset. Linear trends over the whole epoch were removed using the entire epoch to calculate the linear component in all channels. Each epoch was baseline corrected to the $200 \mathrm{~ms}$ preceding stimulus presentation. Horizontal eye movements were detected by a change of $80 \mu \mathrm{V}$ or more in the horizontal EOG channel and the corresponding trials removed from the analysis. As a result one subject's data had to be discarded due to a large number of horizontal eye-movement artefacts ( $>50$ of trials). The resulting artefact-free trials were averaged separately for each condition (eight conditions in total).

The resulting ERPs were then prepared for statistical analysis. Four peaks were identified from the visual ERPs generated in response to cues: P1, N1, P2 and N2 peaks. Of more theoretical interest, a further peak was identified that was generated in response to the target stimuli, which we refer to as $\mathrm{P} 1_{\text {target }}$. Initially, an estimate of the latency of these peaks was determined from the grand average waveform (averaged over all participants and conditions). These values were then used to create a topographic map that was used to identify groups of electrodes showing the greatest amplitudes for that peak (see Fig. 2a). On the basis of this data, four groups of electrodes were selected for further analysis: a left-posterior group (electrodes P5, P7, PO7), a right-posterior group (electrodes P6, P8, PO8), a mid-posterior group (electrodes Cz, CPz, Pz), and an anterior group (electrodes FCz, FC1, FC2). Average ERPs were created for each group of electrodes. Lastly, an algorithm was used to automatically detect the individual peak latencies and amplitudes (i.e. the mean amplitude over $20 \mathrm{~ms}$ centred on the peak latency) for each subject, condition and electrode group evident within $\pm 50 \mathrm{~ms}$ of the grand-average estimated latency of each peak. The amplitude and latency data were then used for statistical analysis.

The main statistical analysis for the purpose of answering the research question examines the modulation of the $\mathrm{P} 1_{\text {target }}$ by the preceding cues. Specifically, we examine the effect of the congruency (in the side of presentation) of the threat cues with the targets on the amplitude of the visual ERP generated in response to the target stimuli ( $\left.\mathrm{P} 1_{\text {target }}\right)$. Within the same analysis, we also investigated the effect of cue type (evolutionary relevant vs. irrelevant). A repeated measures ANOVA was constructed with the factors: electrode group (left-posterior, right-posterior, mid-posterior, 
a

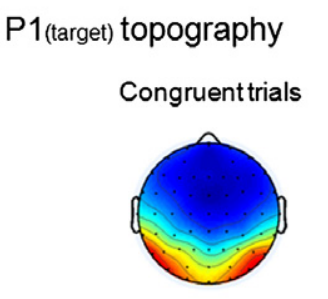

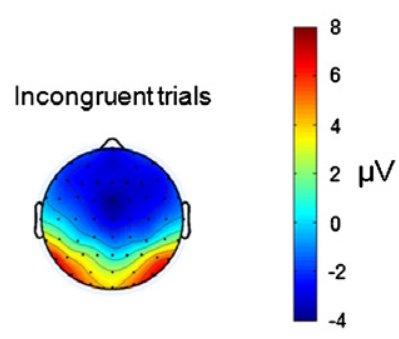

Right posterior electrodes:

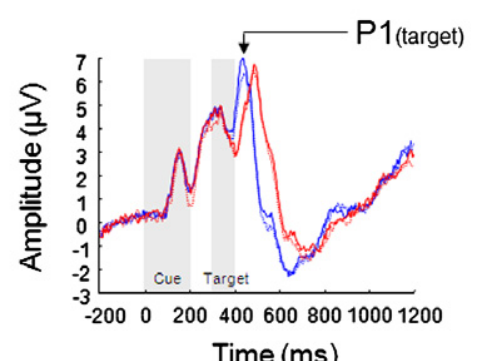

Time (ms)

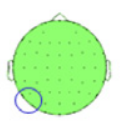

Targets presented in

right visual field

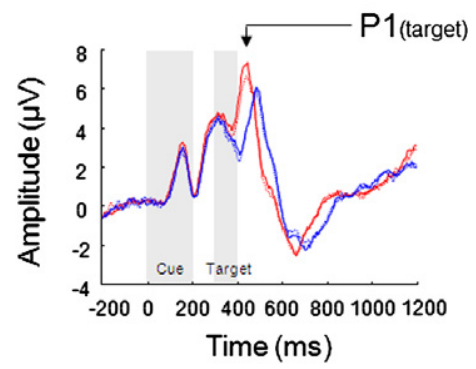

Threat cues congruent with targets

Threat cues incongruent with targets

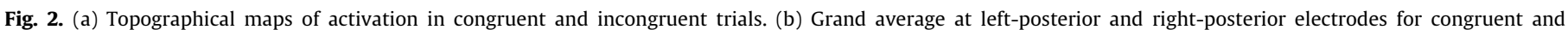
incongruent threatening targets.

anterior), target position (left vs. right), threat cue congruency (congruent vs. incongruent with the target location) and cue type (evolutionarily relevant vs. irrelevant). In order to determine which electrode groups were showing the largest congruency effects, the ANOVA was then repeated for each electrode group individually (four separate ANOVAs in total), each of which considered the three factors of target position (left vs. right), threat cue position (congruent vs. incongruent) and cue type (evolutionary relevant vs. irrelevant). All ANOVA results were Greenhouse-Geisser corrected for non-sphericity. Post-hoc paired-samples $t$-tests were performed to identify which condition effects explained the ANOVA results. A similar ANOVA was conducted on $\mathrm{P} 1_{\text {target }}$ latency.

We do not report statistical analyses for peaks preceding the target (cue P1, N1, P2, and N2). This is because those peaks were not theoretically important, as it is the $\mathrm{P} 1_{\text {target }}$ that indexes the allocation of attention to threat. Furthermore, the fact that the threatening and neutral cues are presented simultaneously prevents a meaningful analysis of these data as a function of threat.

\section{Results}

\subsection{Behavioural data}

The ANOVA comparing average reaction times in $\mathrm{ms}$ revealed a main effect of cue type, $F(1,31)=10.41, p<.01, n_{p}^{2}=.25$, with evolutionary irrelevant stimuli leading to shorter reaction times $(M=618$, $S E=10)$ than evolutionary relevant ones $(M=626, S E=10)$. There was also a main effect of target position, $F(1,31)=20.69, p<.001$, $n_{p}^{2}=.39$, with right-hand responses showing shorter reaction times $(M=611, S E=9)$ than left hand responses $(M=633, S E=11)$. This is likely to be an artefact of the fact that all participants recruited for the study were right-handed. There was no main effect of threat cue position nor did it interact with the location of the target. No other effects were significant.

\subsection{EEG data}

The main analysis explored the effect of congruency of threat cue with target location on the P $1_{\text {target }}$ peak, as a function of evolutionary relevance. In the first ANOVA, in which all electrode groups were considered within the same model, all four factors were found to interact, with a significant electrode group $*$ threat cue congruency $*$ target position $*$ cue type interaction $(p<.007)$, suggesting a complex relationship between threat type and congruency effects that was dependent on the electrode group being considered in addition to the target position. The main effect of cue type was significant $(p<.05)$.

Separate ANOVAs were performed for each electrode group individually. There were main effects of threat cue congruency in left-posterior $(p<.003)$ and right-posterior $(p<.000)$ electrode groups. In both cases, the amplitude of the $\mathrm{P} 1_{\text {target }}$ peak was greater when the target was presented in the same location as the threatening cue compared to the neutral cue.

This effect of congruency however interacted with target location at left-posterior $(p<.007)$ and right-posterior $(p<.001)$ electrode groups. Post-hoc $t$-tests revealed that there was a bigger effect of congruency for targets presented contralaterally to the electrode group being considered (Fig. 2b). Targets presented to the right visual field produced a significant congruency effects at left-posterior $(p<.001)$ but not right-posterior electrodes (Fig. 3). Targets presented to the left visual field produced an effect of congruency for right-posterior $(p<.000)$ electrodes but not left-posterior electrodes. Thus, the threatening cues modulated the amplitude of the $\mathrm{P} 1_{\text {target }}$ peak for contralateral posterior electrodes but not ipsilateral posterior electrodes.

Of interest given the current hypotheses, the effect of congruency did not interact with evolutionary relevance of the cue for left-posterior electrodes, nor was the three way interaction significant. The right-posterior electrode group however showed a 


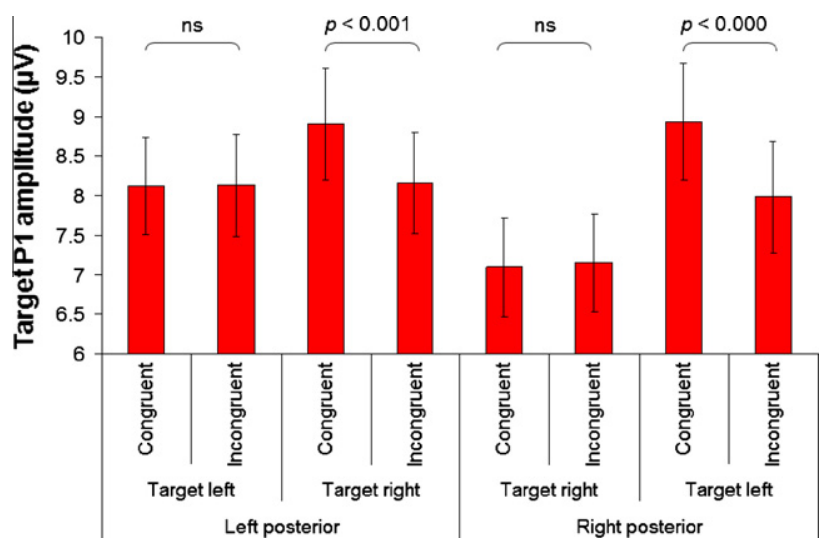

Fig. 3. Effect of threat-target congruency on $\mathrm{P} 1_{\text {target }}$ peak. Bars represent $\mathrm{P} 1_{\text {target }}$ peak amplitude, comparing the effects of the congruency of threat cue with target location at left-posterior and right-posterior electrode groups. Error bars indicate the standard error of the mean.

threat congruency $*$ target position $*$ cue type interaction $(p<.007)$. Post-hoc paired $t$-tests were used to explore these effects by comparing congruent vs. incongruent threat cue positions separately for each threat type (evolutionary relevant, irrelevant) and electrode group (left-posterior, right-posterior) for contralaterally presented targets (as the effect of threat is specific to contralateral presentation). At right-posterior electrodes, processing was facilitated by evolutionarily irrelevant threat cues $(p<.000 ; p<0.001$ after correction for multiple comparisons). Although the difference was in the same direction, this was not significant for evolutionary relevant threats after correcting for multiple comparisons $(p=.96)$ (Fig. 4). Congruent trials also led to a greater $\mathrm{P} 1_{\text {target }}$ amplitude at left-posterior electrodes, although this was not significant after correcting for multiple comparisons for both evolutionarily irrelevant threat cues $(p=.32)$ and evolutionarily relevant threat cues $(p=.40)$. Thus, the comparisons show similar effects of threat cue congruency for evolutionary relevant and irrelevant targets in contralateral electrodes. The interaction at right-posterior electrodes stems from the fact that this effect was somewhat stronger for evolutionarily irrelevant stimuli at right-posterior electrodes only.

We performed the same analyses for mid-posterior and anterior electrodes. None of the effects were significant for mid-posterior electrodes. For anterior electrodes, the main effect of threat con-

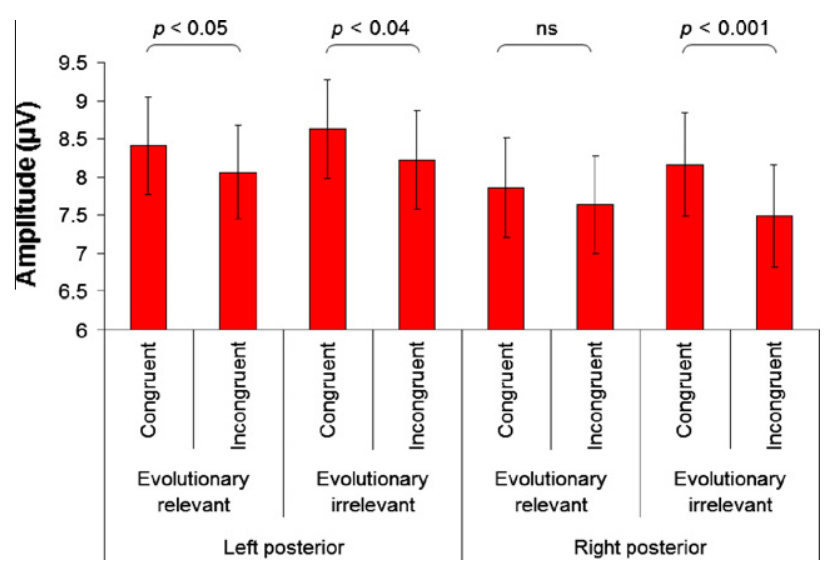

Fig. 4. Interaction between threat-target congruency and stimulus type at $\mathrm{P} 1_{\text {target }}$ peak. Bars represent $\mathrm{P} 1_{\text {target }}$ peak amplitude, comparing the effects of the congruency (congruent vs. incongruent) of threat cues with targets when for evolutionary relevant and irrelevant threats separately. Results are shown for contralateral presentations of targets at left-posterior and right-posterior electrode groups only. Error bars indicate the standard error of the mean. gruency was significant $(p<.05)$ again with greater amplitude when the target followed in the same location as the threat cue. This effect did not interact with evolutionary relevance or with threat location. For mid-posterior electrodes, none of the effects reached significance.

The latency of the P $1_{\text {target }}$ was entered into a similar ANOVA examining the effect of electrode group, target location, cue type and congruency. The only significant effect was a target location $*$ electrode interaction, $F(3,90)=40.91, p<.001, n_{p}^{2}=.58 . \mathrm{P} 1_{\text {tar- }}$ get peak latency was earlier for targets presented to the right for left-posterior electrodes, and for targets presented to the left for right-posterior electrodes, showing an expected contralateral processing superiority. No other effects were significant.

\section{Discussion}

This experiment provides further evidence that the threat-superiority effect is a robust attentional phenomenon. Threatening cues modulated the neural processing of subsequently presented neutral targets. The amplitude of the P1 peak was greater when targets followed in the same location as a threatening cue, suggesting attention was allocated to the location of the threatening cues. This confirms attentional capture by threatening stimuli, and is consistent with the robust threat-superiority effect observed in behavioural, electrophysiological, and functional imaging data. It is also consistent with recent data suggesting a rapid modulation of processing in visual areas by threat (Pourtois, Grandjean, Sander, \& Vuilleumier, 2004; Vuilleumier et al., 2003; Vuilleumier et al., 2004). The effect was found specifically in the contralateral posterior electrodes, consistent with other data showing stronger attentional modulation of the P1 peak in contralateral occipital electrodes (Mangun, Buonocore, Girelli, \& Jha, 1998).

The main novel contribution of this investigation was to compare the modulation of evoked potentials by evolutionarily relevant and irrelevant threats. We found that by and large, both types of threats produced similar effects. Threat cues increased $\mathrm{P} 1_{\text {target }}$ amplitude in contralateral electrodes. This pattern was stronger for evolutionary irrelevant threats, with the pairwise comparisons not reaching significance in the case of evolutionarily relevant threats. In addition to the similar modulation of $\mathrm{P} 1_{\text {target }}$ amplitude, the latency of the $\mathrm{P} 1_{\text {target }}$ peak did not differ across evolutionarily relevant and irrelevant threats. Thus, overall, evolutionarily relevant and irrelevant threats seem to modulate neural processing similarly.

The fact that we found a somewhat larger effect for evolutionarily irrelevant threats must be interpreted with caution. This may be due to the differences in perceptual features between the evolutionarily relevant and irrelevant stimuli. The modern stimuli present simpler shapes, and less complex images overall, which may modulate the level of attentional resources engaged by the processing of the cues and consequently affect the potential to detect significant differences in $\mathrm{P} 1_{\text {target }}$ modulation as a function of threat. This possibility is consistent with the main effect of cue type observed in the overall analysis. Biological stimuli evoked greater amplitudes, regardless of threat value, which is consistent with the idea that they were perceptually more complex and required greater attentional engagement. The behavioural data is also consistent with this account, with evolutionarily relevant stimuli leading to longer reaction times. Unfortunately it is not possible to compare perceptually identical stimuli that are evolutionary relevant and irrelevant. In some of our ongoing work, we are using conditioning procedures to compare neurophysiological processing of perceptually identical threatening and neutral stimuli. However it is not possible to manipulate the evolutionary relevance keeping perceptual features constant using conditioning (or 
any other procedure) as this is intrinsically linked with the identity of the stimulus. This is why in this experiment we focus on interpreting the simple effect of threat for each type of stimuli (evolutionary relevant and irrelevant). Nevertheless, replicating these findings with a different set of stimuli in the future will strengthen conclusions about the robustness of the effect.

Our main conclusion about the similarity between evolutionarily relevant and irrelevant threats is based on the absence of an interaction. Any conclusion based on negative findings must obviously be interpreted with caution. It is possible that our investigation lacked sufficient power to detect existing differences. However, we found a robust effect of threat on $\mathrm{P} 1_{\text {target }}$ amplitude, and a robust effect of evolutionary relevance, independent of threat. We simply did not observe important interactions between the two factors. Thus, if there are differences between the way evolutionarily relevant and irrelevant threats are processed, they may be of minimal functional significance.

The absence of an effect is theoretically important in this case, and our results are consistent with a growing body of work showing the flexibility that characterizes the fear detection system. There are contradictory findings at the moment in the literature concerning the nature of fear processing, and the extent to which it is flexible or constrained. A large body of work presents evidence that fear learning is highly constrained by biological imperatives, with evolutionarily relevant fear targets being either learned more quickly or processed more efficiently (e.g., Cook, Hodes, \& Lang, 1986; Öhman et al., 2001; Tomarken, Mineka, \& Cook, 1989). However, more recent data shows similar effects of prepared and unprepared fear stimuli, even in implicit processing and physiological responses (Brosch \& Sharma, 2005; Flykt, Esteves, \& Öhman, 2007; Olsson, Nearing, \& Phelps, 2007). Although our data will not settle the matter, our results are consistent with the view of fear processing as being at least somewhat flexible.

Obviously, the fact that we did not observe differences in P1 modulation by threat as a function of evolutionary relevance does not rule out the possibility of modular processing of fear stimuli. Different processing of evolutionarily relevant and irrelevant stimuli may occur at another stage of information processing. The P1 monitors early visual processing of the stimuli, and has been shown to be sensitive to threat value. Modular processing of fear might be engaged once threat is detected and identified. Our results show that the early sensory processing of threatening targets is not modulated by evolutionary relevance. Future research will be able to explore other components.

The fact that we observed a threat-superiority effect in the ERP data in the absence of an effect on RTs might seem surprising. However, there are many examples in the literature of studies finding differences in the electrophysiological processing of threat and neutral stimuli in the absence of behavioural differences (BarHaim, Lamy, \& Glickman, 2005; Thomas, Johnstone, \& Gonsalvez, 2007; Weinstein, 1995). This is particularly likely when using unselected samples, as opposed to studies comparing high and low or clinically anxious individuals for instance. It may relate to the fact that ERP measures are more sensitive than behavioural measures. More generally though, the absence of a measure of individual differences in anxiety represents one limitation of the current experiment. Our primary interest was in the basic mechanism as observed in unselected samples but future work may well uncover that these effects are modulated by individual differences in anxiety.

This experiment has certain methodological limitations that should be taken into consideration when interpreting the findings. The main one is the perceptual issues alluded to previously. Because evolutionary significance needs to be examined using different categories of objects, differences in perceptual features of these different object categories may be responsible for differences or absence of differences. In addition, affective differences may also exist. The use of a fixed interval between cue and target is also not ideal and future studies should use variable SOAs to eliminate the possible effect of differential anticipatory eye movements. It is also possible that the images of evolutionary relevant and irrelevant threats evoked different levels of fear. However, the fact that both types of threats significantly modulated the amplitude of evoked potentials to the target suggests these differences may be minimal and do not prevent our conclusions concerning the fact that evolutionarily relevant and irrelevant threatening cues both modulate attentional allocation.

\section{References}

Amaral, D. G., Behniea, H., \& Kelly, J. L. (2003). Topographic organization of projections from the amygdala to the visual cortex in the macaque monkey. Neuroscience, 118, 1099-1120.

Bar-Haim, Y., Lamy, D., \& Glickman, S. (2005). Attentional bias in anxiety: A behavioral and ERP study. Brain and Cognition, 59, 11-22.

Blanchette, I. (2006). Snakes, spiders, guns, and syringes: How specific are evolutionary constraints on the detection of threatening stimuli? The Quarterly Journal of Experimental Psychology, 59, 1484-1504.

Brosch, T., \& Sharma, D. (2005). The role of fear-relevant stimuli in visual search: A comparison of phylogenetic and ontogenetic stimuli. Emotion, 5, 360-364.

Catani, M., Jones, D. K., Donato, R., \& Ffytche, D. H. (2003). Occipito-temporal connections in the human brain. Brain, 126, 2093-2107.

Clark, V. P., \& Hillyard, S. A. (1996). Spatial selective attention affects early extrastriate but not striate components of the visual evoked potential. Journal of Cognitive Neuroscience, 8, 387-402.

Cook, E. W., Hodes, R. L., \& Lang, P. J. (1986). Preparedness and phobia: Effects of stimulus content on human visceral conditioning. Journal of Abnormal Psychology, 95, 195-207.

Eimer, M. (1993). Spatial cueing, sensory gating and selective response preparation. An ERP study on visuospatial orienting. Electroencephalography and Clinical Neurophysiology, 88, 408-420.

Flykt, A., Esteves, F., \& Öhman, A. (2007). Skin conductance responses to masked conditioned stimuli: Phylogenetic/ontogenetic factors versus direction of threat? Biological Psychology, 74, 328-336.

Fox, E., Griggs, L., \& Mouchlianitis, E. (2007). The detection of fear-relevant stimuli: Are guns noticed as quickly as snakes? Emotion, 7, 691-696.

Jung, T., Makeig, S., Westerfield, M., Townsend, J., Courchesne, E., \& Sejnowski, T. (2000). Removal of eye activity artifacts from visual event-related potentials in normal and clinical subjects. Clinical Neurophysiology, 111, 1745-1758.

Lane, R. D., Chua, P. M. L., \& Dolan, R. J. (1999). Common effects of emotional valence, arousal and attention on neural activation during visual processing of pictures. Neuropsychologia, 37, 989-997.

Li, X., Li, X., \& Luo, Y.-J. (2005). Anxiety and attentional bias for threat: An eventrelated potential study. Neuroreport: For Rapid Communication of Neuroscience Research, 16, 1501-1505.

LoBue, V. (2010). What's so scary about needles and knives? Examining the role of experience in threat detection. Cognition and Emotion, 24, 180-187.

Mangun, G. R., Buonocore, M. H., Girelli, M., \& Jha, A. P. (1998). ERP and fMRI measures of visual spatial selective attention. Human Brain Mapping, 6, 383-389.

Öhman, A., Flykt, A., \& Esteves, F. (2001). Emotion drives attention: Detecting the snake in the grass. Journal of Experimental Psychology: General, 130, 466-478.

Öhman, A., Flykt, A., Lundqvist, D., Lane, R. D., \& Nadel, L. (2000). Unconscious emotion: Evolutionary perspectives psychophysiological data and neuropsychological mechanisms. Cognitive neuroscience of emotion. New York, NY US: Oxford University Press.

Öhman, A., Lundqvist, D., \& Esteves, F. (2001). The face in the crowd revisited: A threat advantage with schematic stimuli. Journal of Personality and Social Psychology, 80, 381-396.

Öhman, A., \& Mineka, S. (2001). Fears, phobias, and preparedness: Toward an evolved module of fear and fear learning. Psychological Review, 108, 483-522.

Olsson, A., Nearing, K. I., \& Phelps, E. A. (2007). Learning fears by observing others: The neural systems of social fear transmission. Social Cognitive and Affective Neuroscience, 2, 3-11.

Perchet, C., Revol, O., Fourneret, P., Mauguiere, F., \& Garcia-Larrea, L. (2001). Attention shifts and anticipatory mechanics in hyperactive children: An ERP study using the Posner paradigm. Biological Psychiatry, 50, 44-57.

Perez-Edgar, K., Fox, N. A., Cohn, J. F., \& Kovacs, M. (2006). Behavioral and electrophysiological markers of selective attention in children of parents with a history of depression. Biological Psychiatry, 60, 1131-1138.

Posner, M. I., Walker, J. A., Friedrich, F. J., \& Rafal, R. D. (1984). Effects of parietal injury on covert orienting of attention. Journal of Neuroscience, 4, 1863-1874.

Pourtois, G., Dan, E. S., Grandjean, D., Sander, D., \& Vuilleumier, P. (2005). Enhanced extrastriate visual response to bandpass spatial frequency filtered fearful faces: Time course and topographic evoked-potentials mapping. Human Brain Mapping, 26, 65-79.

Pourtois, G., Grandjean, D., Sander, D., \& Vuilleumier, P. (2004). Electrophysiological correlates of rapid spatial orienting towards fearful faces. Cerebral Cortex, 14, 619-633. 
Pourtois, G., Schwartz, S., Seghier, M. L., Lazeyras, F., \& Vuilleumier, P. (2006). Neura systems for orienting attention to the location of threat signals: an eventrelated fMRI study. Neuroimage, 31, 920-933.

Pourtois, G., Thut, G., De Peralta, R. G., Michel, C., \& Vuilleumier, P. (2005). Two electrophysiological stages of spatial orienting towards fearful faces: Early temporo-parietal activation preceding gain control in extrastriate visual cortex. Neuroimage, 26, 149-163.

Rich, B. A., Schmajuk, M., Perez-Edgar, K. E., Pine, D. S., Fox, N. A., \& Leibenluft, E. (2005). The impact of reward, punishment, and frustration on attention in pediatric bipolar disorder. Biological Psychiatry, 58, 532-539.

Rotshtein, P., Richardson, M. P., Winston, J. S., Kiebel, S. J., Vuilleumier, P., Eimer, M. et al. (2010). Amygdala damage affects event-related potentials for fearful faces at specific time windows. Human Brain Mapping, 31, 1089-1105.

Smith, N. K., Cacioppo, J. T., Larsen, J. T., \& Chartrand, T. L. (2003). May I have your attention, please: Electrocortical responses to positive and negative stimuli. Neuropsychologia, 41, 171-183.

Sugase, Y., Yamane, S., Ueno, S., \& Kawano, K. (1999). Global and fine information coded by single neurons in the temporal visual cortex. Nature, 400, 869-872.
Thomas, S. J., Johnstone, S. J., \& Gonsalvez, C. J. (2007). Event-related potentials during an emotional Stroop task. International Journal of Psychophysiology, 63, 221-231.

Tomarken, A. J., Mineka, S., \& Cook, M. (1989). Fear-relevant selective associations and covariation bias. Journal of Abnormal Psychology, 98, 381-394.

Vuilleumier, P., Armony, J. L., Driver, J., \& Dolan, R. J. (2003). Distinct spatial frequency sensitivities for processing faces and emotional expressions. Nature Neuroscience, 6, 624-631.

Vuilleumier, P., \& Driver, J. (2007). Modulation of visual processing by attention and emotion: Windows on causal interactions between human brain regions. Philosophical Transactions of the Royal Society, 362, 837-855.

Vuilleumier, P. Richardson, M. P. Armony, J. L. Driver, J. \& Dolan, R. J. (2004). Distant influences of amygdala lesion on visual cortical activation during emotional face processing. Nature Neuroscience, 7, 1271-1278.

Weinstein, A. M. (1995). Visual ERPs evidence for enhanced processing of threatening information in anxious university students. Biological Psychiatry, $37,847-858$. 